\title{
Sizing of Spray Particles using Image Processing
}

\section{Technique}

\author{
Sang Yong Lee* and Yu Dong Kim \\ Department of Mechanical Engineering \\ Korea Advanced Institute of Science and Technology \\ Science Town \\ Daejeon 305-701, Korea \\ Tel.: +82-42-869-3026, Fax.: +82-42-869-8207, \\ E-mail: e_hyunny@cais.kaist.ac.kr
}

* Corresponding Author 


\begin{abstract}
The image processing technique is simple and, in principle, can handle particles with various shapes since it is based on direct visualization. Moreover, a wide measurement area can be covered with appropriate optical arrangement. In the present paper, various techniques of image processing for sizing and counting particles are reviewed and recent developments are introduced. Two major subjects are discussed in detail: identification of particles (i.e., boundary detection and pattern recognition) and determination of in-focus criteria. Finally, an overall procedure for image processing of spray particles is suggested.
\end{abstract}

\title{
Introduction
}

Various optical techniques have been being used as non-intrusive method to measure sizes of drops and particles, such as the technique of phase-Doppler particle analysis (PDPA), light scattering method and the image processing technique [1].

The PDPA technique is based on processing of Doppler effect of the Mie scattering signals coming from the particles passing through a measuring volume. Thereby, simultaneous measurement of size and velocity of individual particles is available. Though the fiber optics devices are adopted in the system, however, the optical alignment is still cumbersome and the price of the equipment is rather expensive. Other technique widely being used is the light scattering method based on the Fraunhofer diffraction theory [2]. This technique is relatively simple and also has an advantage of easy alignment. However, this has disadvantages of multiple scattering and vignetting effects [3] that have to be corrected empirically.

The above two techniques are deducing the particle size information from the optical signals scattered from individual or group of particles in the measuring volume assuming that the particles are all spheres. Thus, basically, only the spherical particles should be processed with those techniques for accurate measurement [4]. On the other hand, in principle, various non-spherical particles can be processed through the image processing technique because it is based on direct visualization, and wide application is possible. Moreover, the measurement accuracy of the image processing techniques is relatively insensitive to the optical properties of the particles compared to the other techniques, and the optical alignment is much easier [5]. However, the image processing technique also has shortcomings: a number of image frames have to be processed to get 
the statistically meaningful distributions for a control volume because the data acquisition rate is usually low (i.e., number of spray particles contained in an image frame is usually small) [6]. Also, the accuracy of the results strongly depends on the depth of field criterion $[6,7]$.

The ultimate goal of the image processing research is to identify the objects of interest (spray particles in the present case) and to measure their sizes. More precisely, the in-focus criteria should be determined to decide whether to count or not, and the boundaries of objects should be detected and identified effectively. The particle identification process includes determination of the threshold level for boundary detection, separation of primary particles from agglomerated or overlapped images of particles, and treatment of non-spherical particles. Finally, once the particles are identified, those are sized and counted to give the size distribution.

In the present paper, various image-processing techniques are discussed and compared to each other to find out their merits and demerits in detail; and then recent developments to improve the measurement accuracy and efficiency of the techniques are briefly introduced.

\section{Fundamental System Hardware}

Basically, the system hardware for image processing consists of a light source, a camera and a computer with the accessories as shown in Fig. 1. In most cases, the camera, object and the light source are aligned mostly in line with backward illumination. However, for image processing of fluorescent particles, the technique of side illumination with a laser sheet is often used. Instantaneous light source (flash strobolight) is used to capture the frozen image of spray and the corresponding flow field. Thus the flashing duration should be as short as possible to capture small or highspeed particles. For the camera system, traditional film cameras may be used; however CCD cameras are widely used recently to eliminate the manual scanning process for digitization. Computers are equipped with a time control board and a frame grabber to synchronize signals and store the captured images. The computer is also used to process the stored images to provide the final results.

\section{In-focus Criterion}

The depth-of-field effect is one of the major factors influencing the measurement accuracy. There are two possible error sources caused by the depth-of- 
field effect $[6,7]$. One is the ambiguity in defining the particle boundaries when the particles are placed outside the focal plane. The ambiguity increases as the particles are being located farther from the focal plane. Thus, one should judge whether to count or not from the degree of ambiguity. This has been studied in some detail by Kim and Kim [8] and will be discussed later. Another is the dependency of the depth of field on the particle size. As the particle size becomes smaller, the depth of field drastically decreases, and hence, small particles in a pre-determined measuring volume are more likely to be missed in counting. As a result, the particle size distribution is biased towards larger size region $[9,10]$. Therefore there should be a way to correct this effect by adjusting the depth of field corresponding to the particle size, which will be also introduced later.

As an indicator of the in-focus criteria, either of the following concepts is adopted: the gray-level gradient at the particle boundaries $[5,11]$ or the contrast value between the particles and the background of the image frame $[4,8,12]$. Later, Koh et al. [10] adopted both concepts together by introducing different size ranges for each.

\section{Criterion by Gray-level Gradient}

As a particle is located farther from the focal plane, the gray level at the edge of the particle image changes more gradually as shown in Fig. 2 [10], and the gray-level gradient can be used as the degree of focus of the particles $[11,13]$. In this figure, $z$ denotes the axial distance from the focal plane with the positive value being the direction toward the CCD camera as shown in Fig. 1. Figure 3 illustrates typical graylevel profiles for an in-focus and an out-of-focus particle [11]. The gray-level gradient of the focused particle is steeper than that of the unfocused one. Two gray levels, $I_{2}$ and $I_{3}$, defining a "halo" around the particle can be identified, which correspond to diameters $d_{2}$ and $d_{3}$, respectively. Then the width of the halo $(\Delta d / 2)$ equivalent to $\left(d_{3}\right.$ $\left.d_{2}\right) / 2$ can be used as an index of in-focus criteria [11]. That is, if $\Delta d / 2$ is larger than $H(d)$, a function of the particle diameter obtained through the calibration process, the corresponding particle is eliminated in counting. Figure 4 exhibits the calibration results for various particle diameters ranging from 30 microns to 340 microns. Thus, by using the calibration result with the halo width, actual particle diameters could be predicted from the measured values.

Lecuona et al. [5] also used the concept of gray-level gradient to eliminate the unfocused particles in simultaneous measurement of particle sizes and velocities. There, they defined the in-focus parameter as 


$$
\text { inf }=K \frac{\operatorname{grad}_{\text {max }}}{\operatorname{grad}_{\text {comp }}},
$$

with the following definition:

$$
\operatorname{grad}_{\text {comp }}=\frac{I_{\max }-I_{b a c k}}{d_{p}} .
$$

Here, $K=0.01$ and $d_{\mathrm{p}}$ represents the particle diameter. Also, $I_{\max }$ and $I_{\text {back }}$ are the maximum and background gray levels, respectively. The gray level gradient at the edge of the particles, $\operatorname{grad}_{\max }$, is obtained from the Sobel operators [14]. This parameter is insensitive to the local brightness of the image frame. Figure 5 shows variations of inf with the distance from the focal plane for different aperture numbers $(F)$ and particle (glass sphere) diameters ranging between $0.5 \mathrm{~mm}$ and $3 \mathrm{~mm}$. Based on this calibration curve, critical value of inf for each size can be determined, and the particles with smaller values of inf are considered as out of focus.

\section{Criterion by Value of Contrast}

Kim and Kim [8] performed an extensive study on the image processing technique to size liquid drops less than 30 microns discharged from a fuel injector. Their study includes the determination of the threshold gray level and in-focus criterion. They introduced the concept of the maximum normalized value of contrast $\left(V C_{\max }\right)$ to determine if the drops are in focus or not. With this concept, the depth-of-field correction has been performed for each size of drops. This criterion is known to be effective for sizing small drops, and will be discussed later along with the work by Koh et al. [10].

In the work of Lebrun et al. [12], out-of-focus images are deconvoluted with the assumption of a Gaussian point-spread function, where the spatial parameters increases with the distance from the focal plane. Similar approach has been performed independently by Malot and Blaisot [4]. The intensity (gray level) distribution on CCD plane may be expressed with the following convolution:

$$
I(r)=A(r) * h(r)
$$

where $A(r)=1-\operatorname{circ}(r / d)$ and $d$ is the geometric image radius. Also

$$
h(r)=\frac{8}{\pi \sigma^{2}} \exp \left(\frac{-8 r^{2}}{\sigma^{2}}\right)
$$

which is the Gaussian point spread function (PSF) and $\sigma$ represents the spatial parameter of PSF. The value of $\sigma$ increases as the distance from the focal plane increases. Also the value of $\operatorname{circ}(r / d)$ is unity for $r \leq d$ and zero elsewhere. Also the image contrast is 
defined as

$$
C=\frac{I(\infty)-I(0)}{I(\infty)+I(0)}
$$

where $I(\infty)$ and $I(0)$ are the intensities at $r \rightarrow \infty$ and at the image center, respectively. The image contrasr can be expressed in terms of particle diameter $(d)$ and the degree of focus $(\sigma)$. In their case, the gray-level threshold was set to $I=0.55\{I(\infty)-I(0)\}+I(0)$. Thereby, through the calibration process with particles of known sizes, a relationship between the true value and the measured one could be obtained. According to Lebrun et al. [12] and Malot and Blaisot [4], the dependency of the ratio between the measured and true radius on the image contrast $(C)$ becomes weaker as the value of $C$ approaches unity. This implies that this criterion is not effective to judge the degree of focus for large particles.

\section{Unified In-focus Criterion}

From the above discussions, it can be realized that the criterion of gray-level gradient is mostly applicable to large particles whereas the criterion of contrast value is for small particles. In this view, Koh et al. [10] suggested to adopt both criteria by introducing the applicable particle-size ranges for each case. For the particles smaller than 30 microns, based on their optical arrangement, the normalized value of contrast [8] defined as

$$
V C=\frac{G_{\mathrm{LB}}-G_{\mathrm{OM}}}{G_{\mathrm{LB}}}
$$

was used as an index of the in-focus criterion. Here, $G_{\mathrm{LB}}$ and $G_{\mathrm{OM}}$ represent the local background and the object minimum gray levels, respectively, as shown in Fig. 6. Figure 7 shows the variation of $V C$ with the distance from the focal plane for particle size range of $8-300$ microns. The $V C$ value becomes the largest at or near the focal plane and decreases as becomes unfocused. However, the variation appears gradual with the larger particles. This implies that $V C$ is no longer effective as an index of the degree of focus. In other words, for a large out-of-focus particle, the radius $(d / 2)$ is always greater than the boundary width $(w)$, and the value of $G_{\mathrm{OM}}$ remains unchanged.

For particles larger than 30 microns, the concept of the gradient indicator $(G I)$ was introduced as an index of the in-focus criterion, defined as

$$
G I=\frac{|\nabla G|}{\left[\frac{G_{\max }-G_{\min }}{2 \Delta x}\right]}
$$

which is similar to inf (Eq. (1)) of Lecuona et al. [5]. Here, from Fig. 8, the gray level 
gradient is

$$
|\nabla G(x, y)|=\left[\left(\frac{\partial G}{\partial x}\right)^{2}+\left(\frac{\partial G}{\partial y}\right)^{2}\right]^{\frac{1}{2}}
$$

and, for pixel \#5, the gray level gradients in $x$ and $y$ directions can be expressed with the Sobel operators as follows;

$$
\begin{aligned}
& \frac{\partial G}{\partial x}=\frac{\left(G_{3}+2 G_{6}+G_{9}\right)-\left(G_{1}+2 G_{4}+G_{7}\right)}{8 \Delta x} \\
& \frac{\partial G}{\partial y}=\frac{\left(G_{7}+2 G_{8}+G_{9}\right)-\left(G_{1}+2 G_{2}+G_{3}\right)}{8 \Delta y}
\end{aligned}
$$

As shown in Fig. 9, the values of GI show the maximums at or near the focal plane and decrease as become farther from the focal plane. Thus GI can be used as an index of the degree of focus as well. Figure 10 shows the depth of field variation with the particle size ranging from 10 to 300 microns, mostly covers the fuel spray drops. Here, by introducing two different indices, $V C$ and $G I$, the measurement accuracy could be maintained below ten percent. The relationship between the depth of field and the particle size is very useful because it can be utilized for depth of field correction for a fixed measuring volume [8].

\section{Other Related Works}

The previous methods introduced in the earlier sections are the techniques generally used to resolve the out-of-focus problem. However, there are some other works related to determination of the in-focus criteria $[6,15]$.

Saylor and Jones [15] developed an image-processing algorithm to improve the performance of the rain imaging system (RIS) for raindrop size measurement. In order to secure a sufficient number of drops in a single image frame, the depth of field should be increased. At the same time, increasing of the depth of field causes the increase of the measurement error since the sizes of the unfocused particles appears to be different from the true values. Therefore, in their work, a method to increase the depth of field was proposed without losing the measurement accuracy. With the backward illumination, a bright spot is observed at the center portion of the transparent particles as shown in Fig. 11. The bright spot is the image of the light source, as seen through the drop, and appears sharp when he drop is at the focal plane. (The light source used in their experiment has a rectangular shape.) As the drop is located farther from the focal plane, the spot becomes ambiguous, and Lecuona et al. [5] also noted that the sharpness of the image may be used as an indicator of degree of focus. Saylor and Jones [15] 
showed that, by using glass spheres, parameter $\alpha$ defined as the ratio between the spot diameter and the drop diameter (Fig. 11) depends solely on the distance from the focal plane. However, the sizes of the glass spheres tested were ranging from $4 \mathrm{~mm}$ to $10 \mathrm{~mm}$ and only applicable to large transparent particles such as raindrops.

Nishino et al. [6] used two CCD cameras to obtain stereo images of particles for simultaneous measurement of size and three velocity components of particles in dispersed two-phase flow. The technique developed was capable of sizing $10-500$ microns. As a part of their experiment, the resolution of the depth-of-field effect in particle sizing was considered. They noted that the diameter of small particle (smaller than 40 microns) tends to be overestimated while that of the larger ones (larger than 100 micros) shows the opposite trend.

\section{Particle Identification}

The shape and size information of the particles in an image frame is obtained through the particle identification process. The general procedure is to separate out the particles from the background of the image frame by using the boundary detection algorithms, and then size and shape of the binary image of the particles are measured and recognized by using the pattern recognition algorithms. Particle diameter can be obtained once the number of pixels occupied by the particle image and the scale factor (length/pixel) of the optical system are fixed. The details are discussed in the following sections.

\section{Boundary Detection}

There are two indicators mainly used to detect the particle boundaries: graylevel threshold and gray-level gradient.

Gray-level threshold is the indicator most widely considered in detecting the particle boundaries since it is simple to use $[4,8,10,11,12,15,16,17,18,19,20,21]$. For a given image frame, the portions with the gray level higher than a threshold value are counted as particles and the rest portions are considered as the background. In this method, the number of the detected particles as well as their sizes depends on the threshold level, and care should be taken to decide this value. There are two different ways to decide the threshold gray level; one is taking an appropriate value simply between the gray levels of each particle and the background $[4,8,10,12,20]$ and another is from the gray-level histogram of the image frames $[11,15,18]$. 
As a simple thresholding method, Kim and Kim [8], Kim et al. [20] and Koh et al. [10] have taken the criterion of the threshold value (C), defined as (Fig. 6)

$$
C=\frac{G_{L T}-G_{L B}}{G_{O M}-G_{L B}}
$$

to be 0.5 . However, the value strongly depends on the quality of the image frames and Lebrun et al. [12] and Malot and Blaisot [4] suggested 0.55 and 0.61, respectively, through their own calibration process.

The method using the gray-level histogram has been adopted in the works of Kim [22], Lee et al. [19], Sudheer and Panda [18] and Otsu [21]. Figure 12 shows a typical gray-level histogram of a spray image, in which the gray-level range near the peak value corresponds to the background. The gray levels of the particles are mostly zero and the local peak corresponds to the ordinate of this plot. Therefore, Lee et al. [19] have taken one-half of the gray level corresponding to the peak of the histogram as the threshold value. On the other hand, Kim [22] took $80 \%$ of the intersection of the abscissa and the tangential line at the steepest gradient of the histogram as the threshold value to identify the particles, as illustrated in Fig. 12. More sophisticated approach has been attempted by Otsu [21] and adopted later by Sudheer and Panda [18]. They determined the threshold value based on the zeroth and the first order cumulative momentums from the gray-level histogram. To help illustrating this method, a schematic of the gray-level histogram was given in Fig. 13 along with important parameters. Here, let $\mu_{0}$ and $\mu_{1}$ represent the mean gray levels (first-order cumulative moments) of each gray-level group of the histogram, respectively, divided by a vertical line in the figure. Similarly, let $\omega_{0}$ and $\omega_{1}$ be the numbers of the pixels belong to each gray-level group (i.e., the zeroth-order cumulative moments). Then the line dividing the gray-level group in Fig. 13 would represent the optimal threshold if the value of $\omega_{0} \omega_{1}\left(\mu_{0}-\mu_{1}\right)^{2}$, proportional to the discriminant parameter $\eta$, is the maximum. It has an advantage of avoiding consideration of the local valleys of the histogram by using the integrated values. However, it should be mentioned that the threshold level determined by this method varies with the change of the number concentration of the particles contained in an image frame even though the size distribution remains the same.

Gray-level gradient method is based on the assumption that the gray-level variation is the steepest at the particle boundaries as illustrated in Fig. 14. Figure 14(a) is the original image of a particle, while Fig. 14(c) is its converted image in the plane of the gray-level gradient. Figs. 14(b) and (d) are the plots of the gray level and its gradient corresponding to Figs. 13(a) and (c), respectively. The brightest part of the ring-shaped image in Fig. 14(c) corresponds to the steepest gradient of the gray level. Kim and Lee 
[23] used this method in identifying the primary particles from the images of heavily overlapped particles. From the plot of gray-level gradient, as exemplified by Fig. 14(d), an appropriate threshold value was imposed to recognize the particle boundary. Here, there may be more than one pixel having the gradient greater than this threshold value; and they went through the boundary thinning process down to the 1-pixel thickness. On the other hand, Nishino et al. [6] traced the maximum gradient points along the edge of the particle boundary by curve fitting.

\section{Pattern Recognition and Size Measurement}

Once the particle boundaries are searched and the binary images are obtained, the shape and size of the particles can be identified and measured. The shapes of the particles are either spherical (circular) or non-spherical (non-circular). Besides, the separation of the overlapped particles is performed in this stage.

Most widely used method to measure the particle size is simply finding the equivalent diameter of circle with the same projected area regardless of its original shape. $[11,12,18]$ For spherical particles, the radius can be obtained from the mean distance from the boundary pixels to the mass center as follows:

$$
\begin{aligned}
& \text { Radius }=\frac{1}{N} \sum_{k=1}^{N} \sqrt{\left(x_{k}-x_{c}\right)^{2}+\left(y_{k}-y_{c}\right)^{2}} \\
& \text { Center }\left(x_{c}, y_{c}\right)=\left(\frac{1}{N} \sum_{k=1}^{N} x_{k}, \frac{1}{N} \sum_{k=1}^{N} y_{k}\right)
\end{aligned}
$$

Here, $N, x_{k}$ and $y_{k}$ denote the number of pixels at the image boundary and their $x-y$ locations in the plane, respectively. The result is usually acceptable because the particles are spherical or nearly spherical. However, it gives inaccurate results for the partly detected particles or overlapped images of particles. To overcome this difficulty, Kim et al. [20] proposed to use the circumcenter of the particle image instead of the mass center. Figure 15 illustrates the concept of this method. Once points $A, B$, and $C$ are selected from the boundary pixels, a triangle $A B C$ is determined as in Fig. 15(a). Then the circumcenter (point $O$ ) of this triangle is located at the cross point of the perpendicular lines $(O P, O Q$ and $O R$ ) bisecting each side. When there are more than three data points at the particle boundary, a circle that best fits those points should be found. That is, with $N$ data points, $N(N-1)(N-2) / 6$ triangles and their circumcenters are found, and from the arithmetic mean values of the center locations and the radii of the circles, a circle that 
best fits all data points can be determined. Here, it should be noted that the three data points should be distant from each other to avoid an erroneous result as illustrated by points $D, E$ and $F$ in Fig. 15(a), and the details on this technique are explained in the work of Kim et al. [20]. The circumcenter-based concept has the advantage when only the partial data points are available for analysis as shown in Fig. 15(b).

An image processing method based on the correlation analysis in the frequency (Fourier) domain has been reported by Cruvinel et al. [17]. First, reference (standard) images of a particle with various sizes are prepared. Then the particles in the image frames of interest were sought by matching their patterns with the reference images by using the Fourier transform technique. Figure 16 illustrates this technique in detail. Figure 16(a) shows an input image treated with a threshold area processing. Figure 16(b) shows the image of a particle to be recognized from the input image. Figures 16(c) and (d) are the Fourier spectra of the input image and the standard image. Figure 16(e) is the correlation map of the spectra obtained through convolution, where the particle of interest shows the highest correlation (i.e., the brightest spot appears), and Fig. 16(f) is the final result recognizing the particle image having the same radius with the standard image.

For overlapped particle images, Lecuona et al. [5], Malot and Blaisot [4], Kim and Lee [24] simply eliminated them since they are considered either odd-shaped foreign materials or erroneously detected. This concept is acceptable only when the drop area fraction (DAF) of the image frame is low since the chances of the particle overlap are usually small. Shen et al. [25] and Zhang and Ishii [26] reported their works on the treatment of the overlapped particles, but the details are not given in their paper. Thus, in the present review, the methods of the particle separation using the Hough transform [16, 23, 27], the boundary curvature detection [28], and the convex-hull method [20] are discussed.

Using of the Hough transform is very effective way to handle the heavily overlapped particles. Basically, the primary particles are separated out from the agglomerated image, and at the same time, each particle size can be estimated. Figure 17 illustrates the principle of the Hough transform process to identify a circle image. The rectangular symbols in Fig. 17(a) (except for pixels $a$, $e$ and $f$ ) are the boundary pixels of a particle to be identified. Circle $A$ is the true circle with radius $R$ and the center is located at $a(X, Y)$. With the assumed radius $(R)$ of circle $A$, its center should exist somewhere at distance $R$ from the boundary pixels. For example, a point of circle $B$ ( $b$-centered circle) with radius $R$ should be the center of circle $A$. Similarly, a point of circle $C$ and $D$, corresponding to boundary pixels $c$ and $d$ should be the center of circle 
$A$. If the same procedure is repeated along the boundary pixels of circle $A$, pixel $a$ will have the highest frequency of pass by the circles. In other words, pixel $a$ has the highest possibility of being the center, and it is recognized as the true center of circle $A$. In practice, the radius of circle $A$ is unknown at the beginning, and the overall procedure should be repeated for various values of $R$. Thus, in parametric space with $x$-, $y$ - and $r$ axes, point $(X, Y, R)$ shows the maximum value(possibility). Figure 17(b) illustrates the possibility of being the center in the parameter space with radius $R$ for the boundary pixels of circle $A$. Here, the possibility is expressed as the brightness, and the possibility viewed to be the highest at the center that corresponds to pixel $a(X, Y)$. However, the Hough transform itself is a time consuming process, and Kruis et al. [27] proposed the sparse Hough transform to resolve this problem. In applying the Hough transform to separate out and size the primary particles of the heavily overlapped image, the centersearching process was improved by Kim and Lee [23]. Crida and Jager [16] used the Hough transform to perform online measurement of rock size distribution. To identify existence of the rocks in the image frame, it was assumed that the rock shape could be modeled using an ellipse with a reasonable accuracy. Also, due to the multi-scale nature of the rocks, multiple levels of image scale were used. However, it takes much longer to process the ellipses (compared to the circles) since there are more parameters to be considered; hence, only one size of the rocks was detected at a particular level of the image scale to reduce the number of parameters in the parametric space.

In principle, primary particles in the overlapped image can be separated out and sized by examining the boundary curvature. Figure 18 shows the basic concept of this approach proposed by Kim et al. [28]. For a circle with its radius $R$, as shown in Fig. $18(\mathrm{a})$, the boundary curvature $(\Delta \theta / \Delta l)$ remains unchanged along the periphery $l$, i.e., $\Delta \theta / \Delta l=1 / R$. For an ellipse, the boundary curvature varies along the periphery with a sinusoidal shape as shown in Fig. 18(b). On the other hand, as depicted in Fig. 18(c), there are abrupt changes of the boundary curvature at the contact points of the overlapped-particle image, and this technique is very useful in handling the overlapped particles. However, since the pixel size is not infinitesimally small, a piecewise averaging technique is needed to smooth out the saw-toothed boundary, and the details can be found from the work of Kim et al. [28].

The methods of Hough transform and boundary-curvature detection are capable of sizing primary particles as well as separating them out of the agglomerated images of particles. However, there is another technique proposed by Kim et al. [20] simply to separate the primary particles. For the overlapped image of two particles, as shown in 
Fig. 19, the hull of the convex polygon can be determined to encompass the overlapped image with the shortest perimeter. Mostly, the distances between the adjacent pixels at the hull of the polygon appear approximately the same except for the line segments connecting two particles. The concave parts of the overlapped image exist underneath those long line segments, and the farthest points from the segments (i.e., points $a$ and $b$ ) are considered as the contact points of two circles. Thus, the line $A B$ connecting points $a$ and $b$ is the dividing line between two particles. This technique is very useful in handling weakly overlapped images because the process time is much shorter than the previously discussed techniques, such as the Hough transform or the boundary curvature examination. Once the primary particles are separated out, each size can be measured by finding their circumcenter as already discussed.

In order to describe the shape of the particle images, the concept of shape factor is widely adopted. The shape factor is defined as perimeter ${ }^{2} /$ area [11] indicating the deviation of the particle image from a circular shape. For a circle, this value is $4 \pi$, and if a particle is not circular this value becomes significantly greater. As a similar concept, Sudheer and Panda [18] have used compactness, which is the reciprocal of the shape factor defined by Fantini et al. [11]. On the other hand, Lecuona et al. [5] have used the combination of circularity and convexity. The circularity is defined as $4 \pi A / P^{2}$, similar to the shape factor and the convexity is the ratio between the convex hull and the perimeter of the object. They considered the images having the values of circularity and convexity lower than 0.8 and 0.95 , respectively, are the overlapped particles or not the ones under consideration, and those should be separated or rejected in counting. On the other hand, Malot and Blaisot [4] introduced the concept of the shape parameter $S_{\mathrm{p}}$, defined as the ratio between the non-common area and the circle as shown in Fig. 20. The value of the shape parameter ranges from 0 to 2 , where the zero value corresponds to a circular $2 \mathrm{D}$ projection. Thus, in their work, the images of drops with a large shape parameter were eliminated.

\section{Other Developments}

Other than the in-focus criteria and the particle identification procedure discussed in the previous part of this paper, some important developments related to the image processing algorithms are introduced in this section.

Kim and Kim [8] divided the pattern recognition process into two major steps. The first step is to identify each drop images in the entire image frame through the 
global processing. Then the entire image frame is divided into subframe windows with $32 \times 32$ pixels each to calculate accurate threshold levels. Sudheer and Panda [18] have used the similar segmentation technique to process the photographs of sprinkler drops in flight. Kim et al. [20] improved the concept of segmentation by Kim and Kim [8]. They performed the local process (in addition to the global process performed by Kim and Kim [8]) for each subframe window. The local process is specific to each subframe window, which is determined by the center and the radius of each image recognized as a single particle (or object) through the global processing of the entire image frame; thereby the threshold level for each subframe can be determined differently for more accurate measurement. In other words, the concept of the local process was proposed to reduce the measurement error by taking account of unevenness of the light illumination on the spray particles.

The concept of the two-stage process of Crida and Jager [16] for measurement of the rocks (solid particles) is somewhat similar to the two-step approach of Kim et al. [20]. This concept is denoted as the human visual system because the algorithm simulates the stage of preattentive vision followed by the attention focusing process, as performed by the human beings. The purpose of the preattentive vision stage is to detect target features for further analysis in the next stage, and the Hough transform is used assuming the particles are in elliptical shape. Then, in the attention focusing stage, target features are re-examined to determine the extent of the object in the image using more sophisticated process.

Another novel approach is the image processing with the laser fluorescence method. Carlos [29] analyzed images of water spray with fluorescent additives using a pulsed laser sheet. Image frames were captured by a camera viewed perpendicular to the laser sheet. This method is capable of wide-field analysis of the instantaneous spray behavior because intensity of light emission from each fluorescent drop is much stronger than the light intensity obtained by backward illumination. Also, the out-offocus problem is considered relatively minor with this method because the thin laser sheet was used as the light source. This technique is extendable to the PIV method for simultaneous measurement of drop sizes and velocities in the field of interest.

Park et al. [30] obtained SMD of gasoline spray by using the principles of laserinduced fluorescence (LIF) and the Mie scattering of drops. The intensities of the LIF $\left(I_{\mathrm{LIF}}\right)$ and Mie $\left(I_{\mathrm{Mie}}\right)$ scattering are proportional to the cube and the square of the drop diameter, respectively. Thus the ratio between two intensities $\left(I_{\mathrm{LIF}} / I_{\mathrm{Mie}}\right)$ should be proportional to SMD of the spray, and the proportionality constant could be obtained from the experiments. 


\section{Overall Procedure for Image Processing of Spray Particles}

In the previous sections, various details on determination of in-focus criteria and identification of spray particles have been introduced. In this section, the overall procedure of image processing utilizing those techniques is discussed. Table 1 illustrates the overall procedure to process an image frame containing spray particles based on the algorithm proposed by Kim [22]. The entire procedure consists of two major steps, namely, the global process (steps $1-2$ ) and the local process (steps 3 -9). Already explained before, this approach is advantageous because the problem of the unevenness of the illumination intensity on the entire image frame can be eliminated.

In the global process, first of all, a threshold level should be determined for the entire image frame and through the boundary detection process (step 1), the original image is changed to the binary one and then the particle boundaries are sought. In this step, either the gray level $[19,22]$ or the gray-level gradient [23] of the particle image may be used as the threshold criterion. In the next step, the approximate radii and the locations of the particles or 'particle-like' objects (such as the overlapped particles and the odd-shaped materials) are identified by searching the their centers using Eqs. (11) and (12).

In the second stage, the local process starts with step 3, dividing the entire image frame into local windows each covering a single particle or a 'particle-like' object. Then, in step 4, the boundary of the object in each local window is sought. Similar to step 1 of the global process, either the gray-level threshold $[4,8,10,12,20]$ or the gray-level gradient threshold $[6,23]$ may be used. Once the object boundaries are detected, primary particles are separated out from the overlapped images [20, 23, 27 , 28] and the odd-shaped materials are filtered out [24], if there are any, as in steps 5 and 6. In the next step (step 7), the out-of-focus particles, which have ambiguous boundaries, are eliminated using the in-focus criterion $[4,5,8,10,11,12]$. Then, in step 8 , the depth-of-field correction [10] is performed to take account of different field depths of each size-group contained in a fixed measuring volume. Finally (step 9), the particles recognized as the correct ones in the image frame are counted and sized for analysis.

\section{Conclusions and Suggestions}

In this work, recent development of the image processing technique to size particles was introduced, which is essentially important in the field of spray/atomization technology. Basically, there are two main issues to be resolved. One is the out-of-focus problem and another is the method of particle identification from the focused images. 
The out-of-focus particles are the largest error sources in counting and sizing them in a measuring volume. Therefore most of the works were concentrated on the establishment of the in-focus criteria. The gray level gradient and the value of contrast were introduced as the indicators of the degree of focus. Each indicator is suitable to different size ranges: the gray level gradient for large particles and the value of contrast for small particles. To cover those size ranges simultaneously, a unified in-focus criterion has been proposed.

Pattern recognition and size measurement are the main subjects of the particle identification. In most cases, particle diameters can be obtained from the projected area of the particles. To process the partially detected or overlapped particles, the convexhull method, Hough transform, pattern matching through Fourier transform, and the boundary-curvature detection method have been developed. To represent the noncircularity of the particle images, various shape factors were introduced.

Improvement of the image processing technique was always possible with the rapid progress in computing hardware. Most of the basic concepts regarding the image processing of the spray drops/particles are established so far. However, there are several important problems still to be resolved. Handling of non-spherical particles is one of them since there are various shapes other than spheres (circles) or ovals (ellipses). Systematic classification of the particle shape should be performed prior to process them. Another is the dependency of the measured results on the system hardware. Determination of the in-focus criteria and the threshold levels depends strongly on the optical arrangement (i.e., optical parameters, such as focal length, aperture size, etc.). Hence, especially for real-time measurement of spray drops, there should be a general (or a standard) procedure to match the optical parameters and the image-processing algorithm to get consistent results. Finally, considering that the ultimate goal of the image processing technique is to recognize the object as the human vision, introduction of the concepts of the artificial intelligence and the neural network may be another break through in this field.

\section{Acknowledgements}

This work has been supported by the Combustion Engineering Research Center (CERC) and the National Research Laboratory (NRL, 99-N-NL-01-C-022) Programs, and partly by the Brain Korea 21 Project. 


\section{References}

[1] N. Chigier, 1983, "Drop Size and Velocity Instrumentation," Prog. Energy Combust. Sci., Vol. 9, pp. 155-177.

[2] J. Swithenbank, J. Beer, D. S. Taylor, D. Abbot and G. C. McGreath, 1977, “A Laser Diagnostic Technique for the Measurement of Droplet and Particle Size Distributions," Prog. Aeronaut. Astronaut., Vol. 53, pp.421-427.

[3] P. N. Wild and J. Swithenbank, 1986, "Beam Stop and Vignetting Effects in Particle Size Measurements by Laser Diffraction," Applied Optics, No. 25, pp. 3520-3526.

[4] H. Malot and J. B. Blaisot, 2000, "Droplet Size Distribution and Sphericity Measurements of Low-Density Sprays through Image Analysis," Part. Part. Syst. Charact., No. 17, pp. 146-158.

[5] A. Lecuona, P. A. Sosa, P. A. Rodriguez and R. I. Zequeira, 2000, "Volumetric Characterization of Dispersed Two-phase Flows by Digital Image Analysis," Measurement Science and Technology, No. 11, pp. 1152-1161.

[6] K. Nishino, H. Kato and K. Torii, 2000, "Stereo Imaging for Simultaneous Measurement of Size and Velocity of Particles in Dispersed Two-Phase Flow," Measurement Science and Technology, No. 11, pp. 633-645.

[7] N. Chigier, 1991, "Optical Imaging of Sprays," Prog. Energy Combustion Sci, No. 17, pp. 211-262.

[8] K. S. Kim and S. S. Kim, 1994, "Drop Sizing and Depth-of-Field Correction in TV Imaging," Atomization and Sprays, No. 4, pp. 65-78.

[9] L. M. Oberdier, 1984, “An Instrumentation System to Automate the Analysis of Fuel-Spray Images using Computer Vision,” ASTM, No. 848, pp. 123-136.

[10] K. U. Koh, J. Y. Kim and S. Y. Lee, 2001, "Determination of In-focus Criteria and Depth of Field in Image Processing of Spray Particles," Atomization and Sprays, Vol. 11, No. 4, pp. 317-333.

[11] E. Fantini, L. Tognotti and A. Tonazzini, 1990, "Drop Size Distribution in Sprays by Image Processing," Computers chem. Engng, Vol. 14, No. 11, pp. 1201-1211.

[12] D. Lebrun, C. E. Touil and C. Özkul, 1996, "Methods for the Deconvolution of Defocused-Image Pairs Recorded Separately on Two CCD Cameras: Application to Particle Sizing," Applied Optics, Vol. 35, No. 32, pp. 6375-6381.

[13] C. Ramshaw, 1968, "A Technique for Drop Size Measurements by Direct Photography and Electronic Image Size Analysis,” J. Inst. Fuel, No. 41, 288-292.

[14] R. C. Gonzalez and R. E. Woods, 1993, Digital Image Processing, Addison Wesley. 
[15] J. R. Saylor and B. K. Jones, 2002, "A Method for Increasing Depth of Field during Droplet Imaging," Review of Scientific Instruments, Vol. 73, No. 6, pp. 2422-2427.

[16] R. C. Crida and G. de Jager, 1997, "An Approach to Rock Size Measurement based on a Model of the Human Visual System," Minerals Engineering, Vol. 10, No. 10, pp. 1085-1093.

[17] P. E. Cruvinel, S. R. Vieira, S. Crestana, E. R. Minatel, M. L. Mucheroni and A. T. Neto, 1999, "Image Processing in Automated Measurements of Raindrop Size and Distribution," Computers and Electronics in Agriculture, Vol. 23, pp. 205217.

[18] K. P. Sudheer and R. K. Panda, 2000, "Digital Image Processing for Determining Drop Sizes from Irrigation Spray Nozzles,” Agricultural Water Management, No. 45, pp. 159-167.

[19] S. Y. Lee, B. S. Park and I. G. Kim, 1991, "Gray Level Factors Used in Image Processing of Two-Dimensional Drop Images," Atomization and Sprays, Vol. 1, No. 4, pp. 389-400.

[20] J. Y. Kim, J. H. Chu and S. Y. Lee, 1999, "Improvement of Pattern Recognition Algorithm for Drop Size Measurement," Atomization and Sprays, Vol. 9, No. 3, pp. 313-329.

[21] N. Otsu, 1979, "A Threshold Selection Method from Gray Level Histogram," IEEE Transactions on Systems, Man and Cybernetics, Vol. SMC-9, No. 1, pp. 6266.

[22] J. Y. Kim, 2000, "Study of Internal Flow Characteristics and Atomization Performance of Effervescent Atomizers," Ph. D Thesis, KAIST, Korea.

[23] Y. D. Kim and S. Y. Lee, 2002, "Application of Hough Transform to Image Processing of Heavily Overlapped Particles with Spherical Shapes," Atomization and Sprays, Vol. 12, No. 4, pp. 451-461.

[24] I. G. Kim and S. Y. Lee, 1990, “A Simple Technique for Sizing and Counting of Spray Drops Using Digital Image Processing,” Exp. Thermal Fluid Sci., Vol. 3, pp. 214-221.

[25] L. Shen, X. Song, Y. Murai, M. Iguchi and F. Yamamoto, 2001, "Velocity and Size Measurement of Falling Particles with Fuzzy PTV," Flow Measurement and Instrumentation, Vol. 12, pp. 191-199.

[26] G. J. Zhang and M. Ishii, 1995, "Isokinetic Sampling Probe and Image Processing System for Droplet Size Measurement in Two-Phase Flow," Int. J. Heat Mass Transfer, Vol. 38, No. 11, pp. 2019-2027. 
[27] F. E. Kruis, J. V. Denderen, H. Burrman and B. Scarlett, 1994, "Characterization of Agglomerated and Aggregated Aerosol Particles Using Image Analysis," Part. Part. Syst. Charact., Vol. 11, pp. 426-435.

[28] Y. D. Kim, S. Y. Lee and J. H. Chu, 2001, "Separation of Overlapped Particles Using Boundary Curvature Information," Proceedings of the 6th Annual Conference on Liquid Atomization and Spray Systems (ILASS-Asia 2001), pp. 259-264.

[29] J. G. Carlos, 2002, "Heat Transfer in Two-Phase Stagnation Point Flow," M. S. Thesis, The University of Michigan, USA.

[30] S. Park, H. Cho, I Yoon and K. Min, 2002, "Measurement of Droplet Size Distribution of Gasoline Direct Injection Spray by Droplet Generator and Planar Image Technique,” Measurement Science and Technology, Vol. 13, pp. 859-864. 


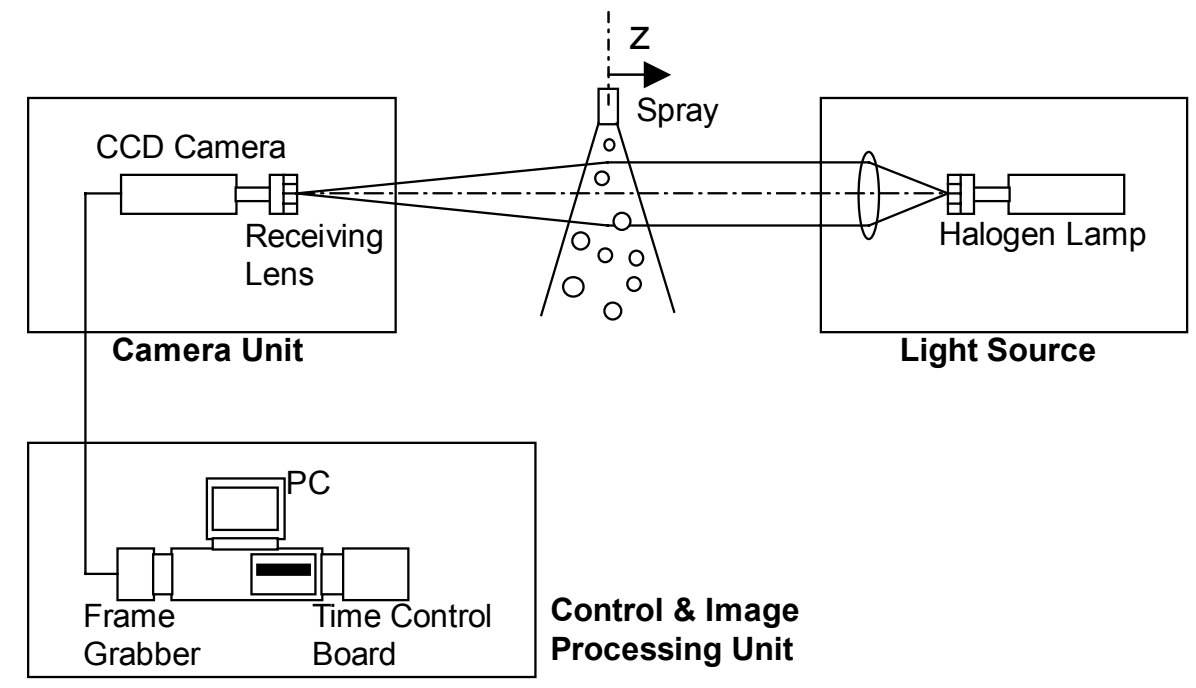

Fig. 1 Hardware setup of image processing technique [22]

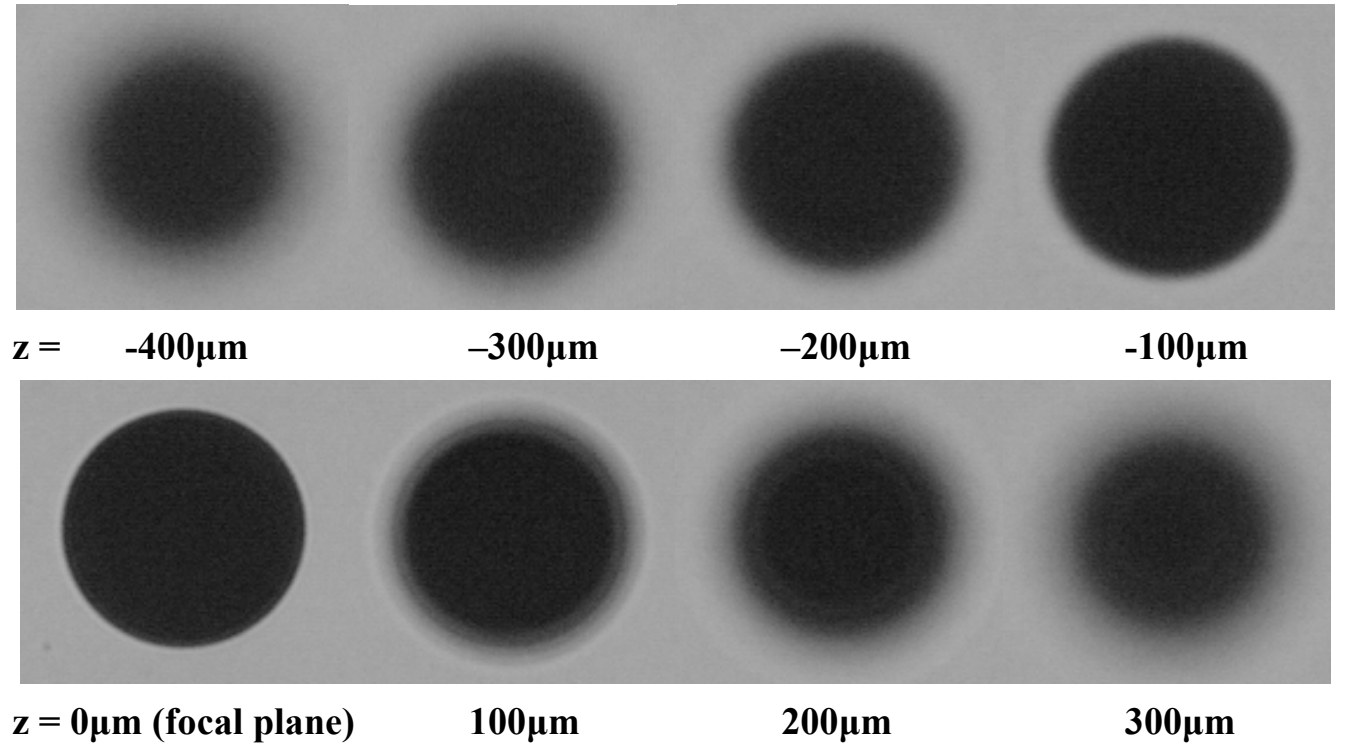

Fig. 2 Change of particle images with distance from the focal plane (300-micron particle) 


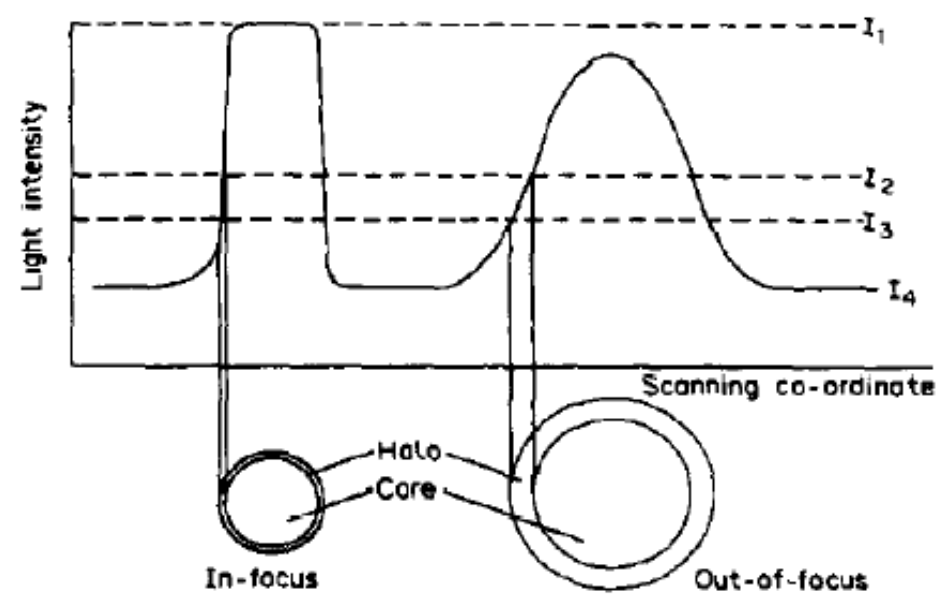

Fig. 3 Drop image intensity profiles [11]

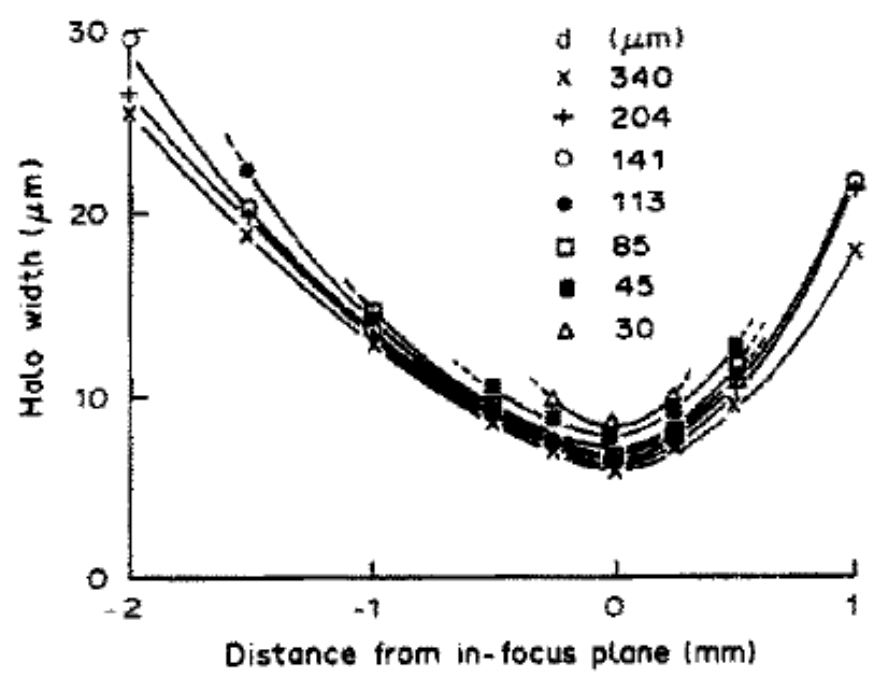

Fig. 4 Halo width variation VS position in field [11] 


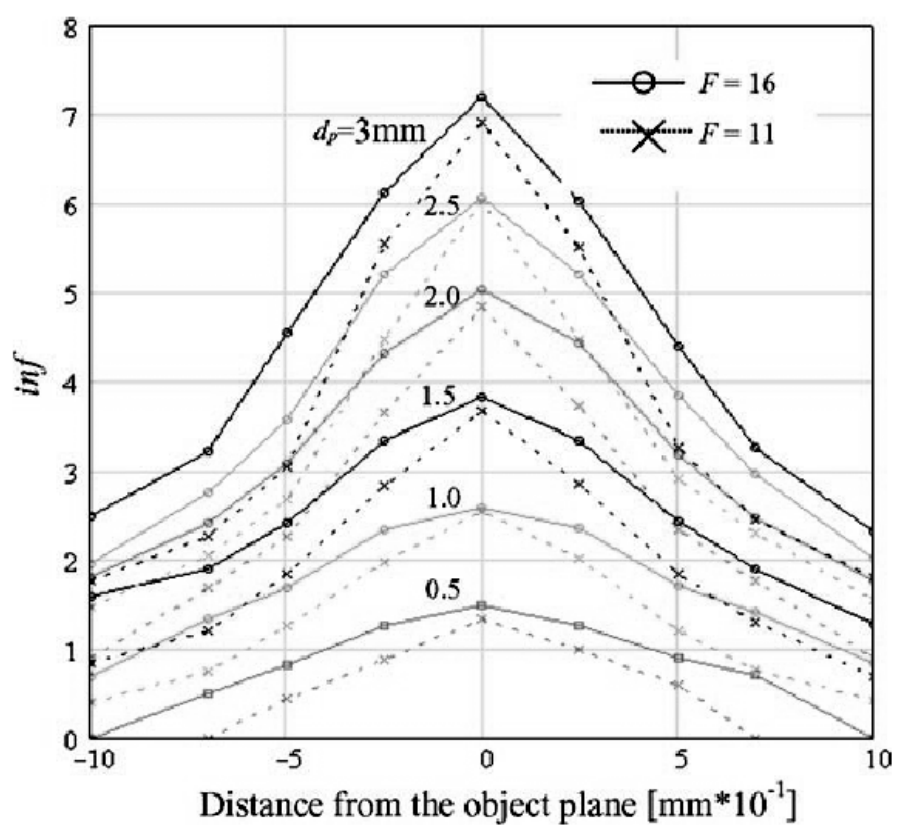

Fig. 5 Variation of inf with respect to axial distance from object plane [5]

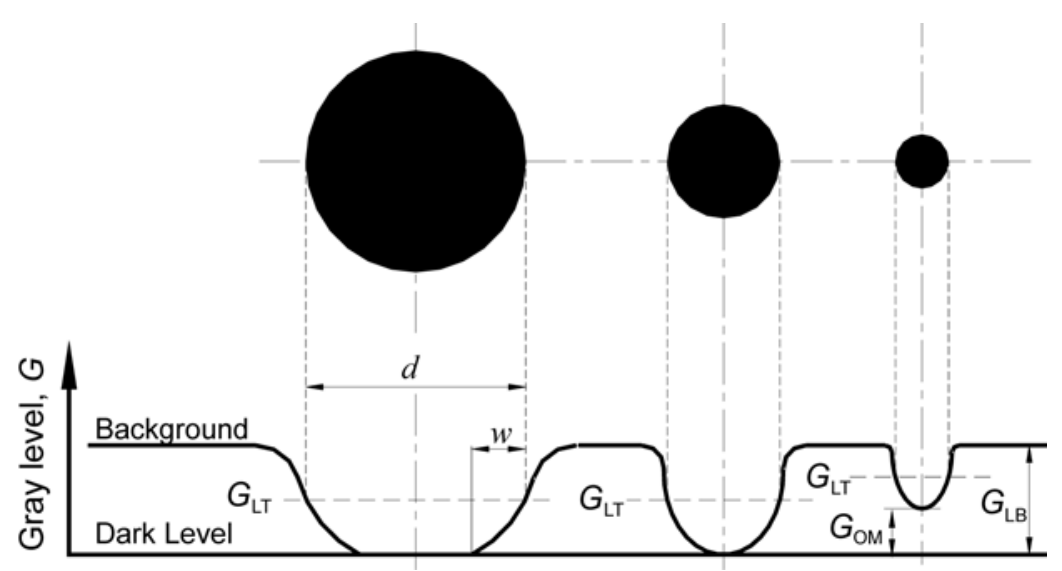

Fig. 6 Gray level changes with different particle size [10] 


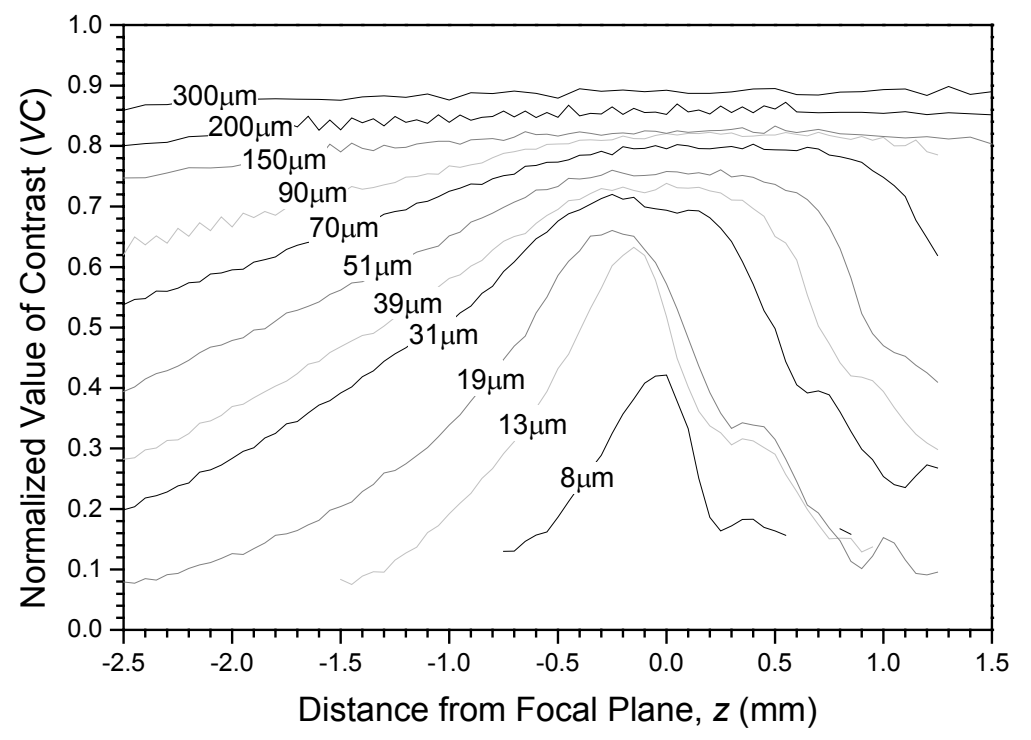

Fig. 7 Variation of normalized value of contrast $(V C)$ with distance from focal plane for each particle size [10]

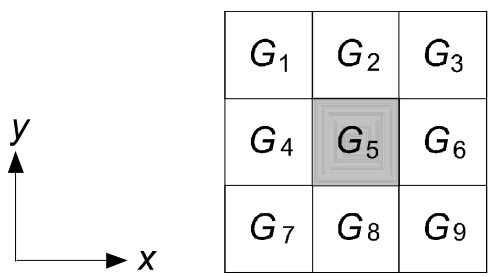

Fig. 8 Numbering of the pixels to express gray level gradient at pixel \#5 


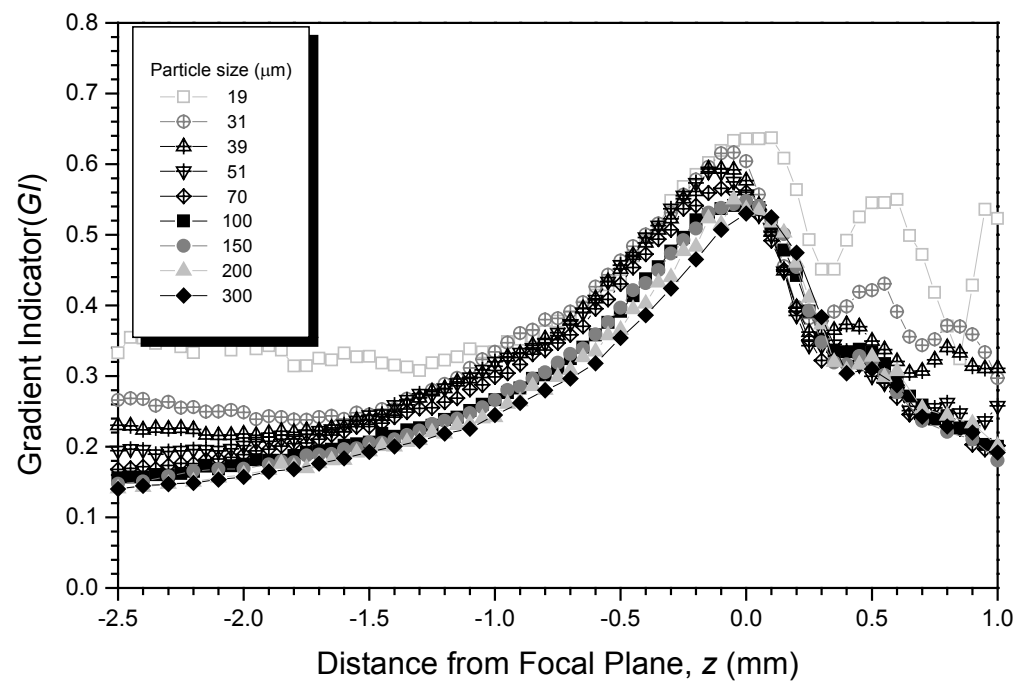

Fig. 9 Variation of gradient indicator(GI) with distance from focal plane for each particle size [10]

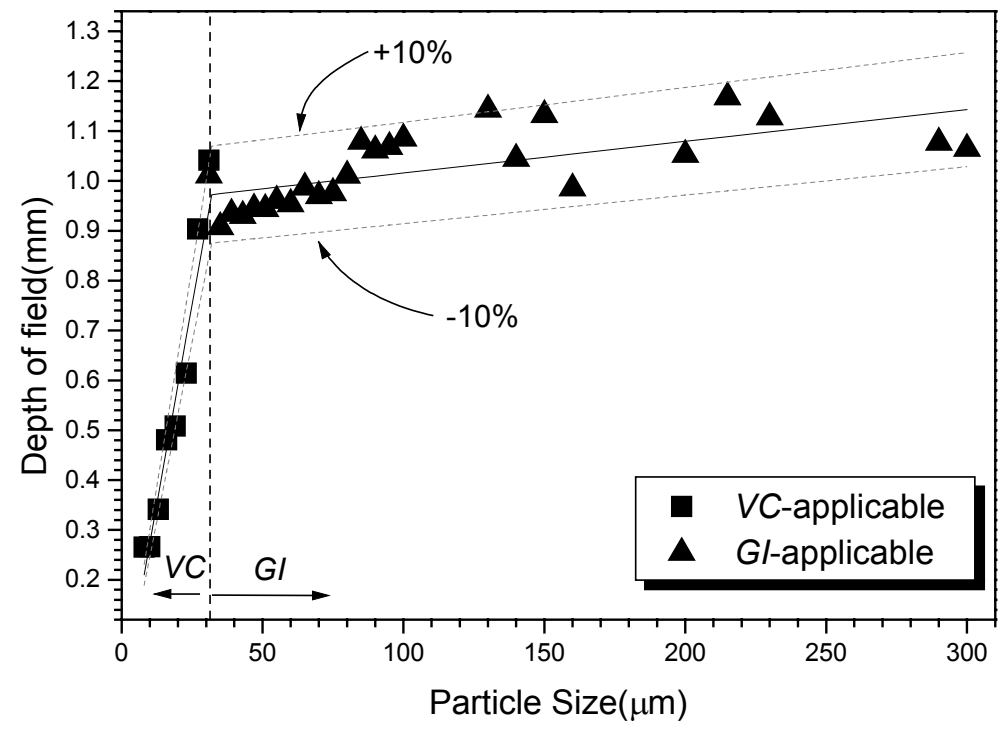

Fig. 10 Variation of depth of field with particle size [10] 


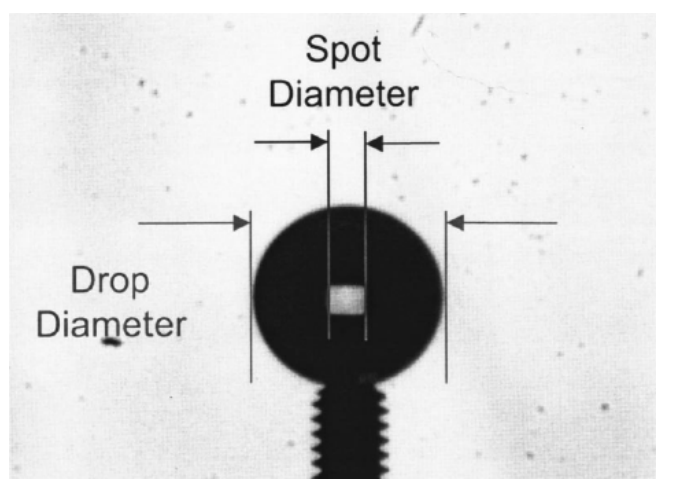

Fig. 11 Sample Image of Glass Sphere [15]

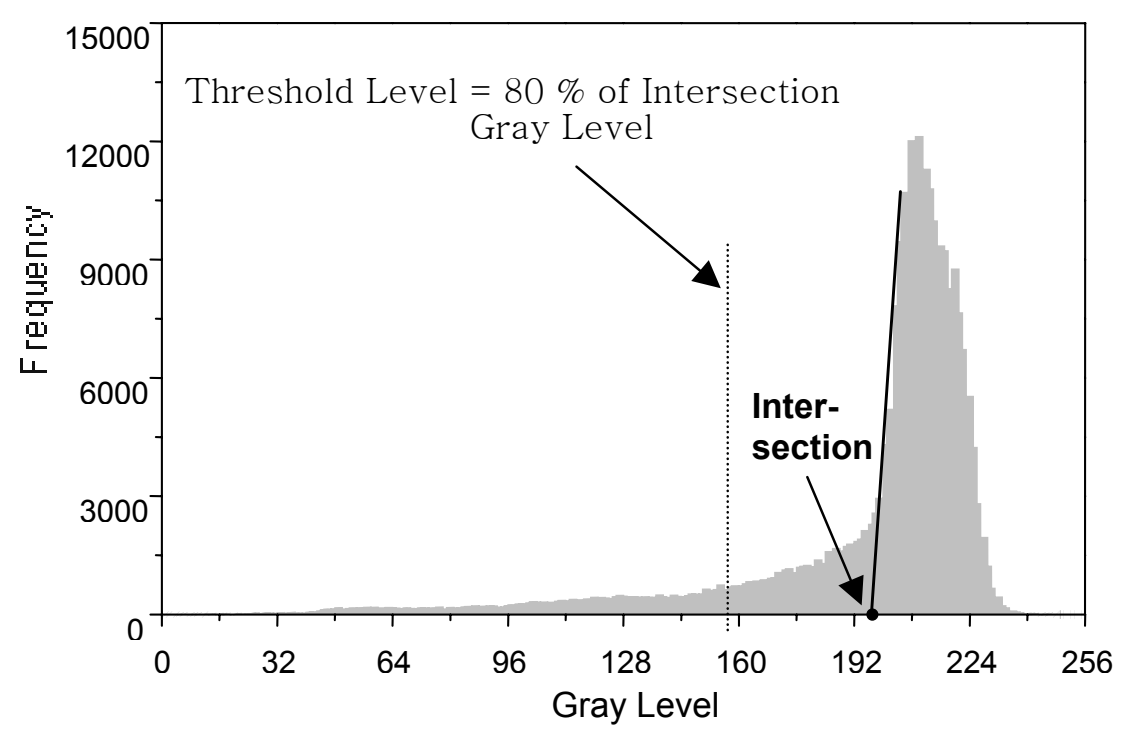

Fig. 12 Typical histogram of a spray image and determination of threshold level [22] 


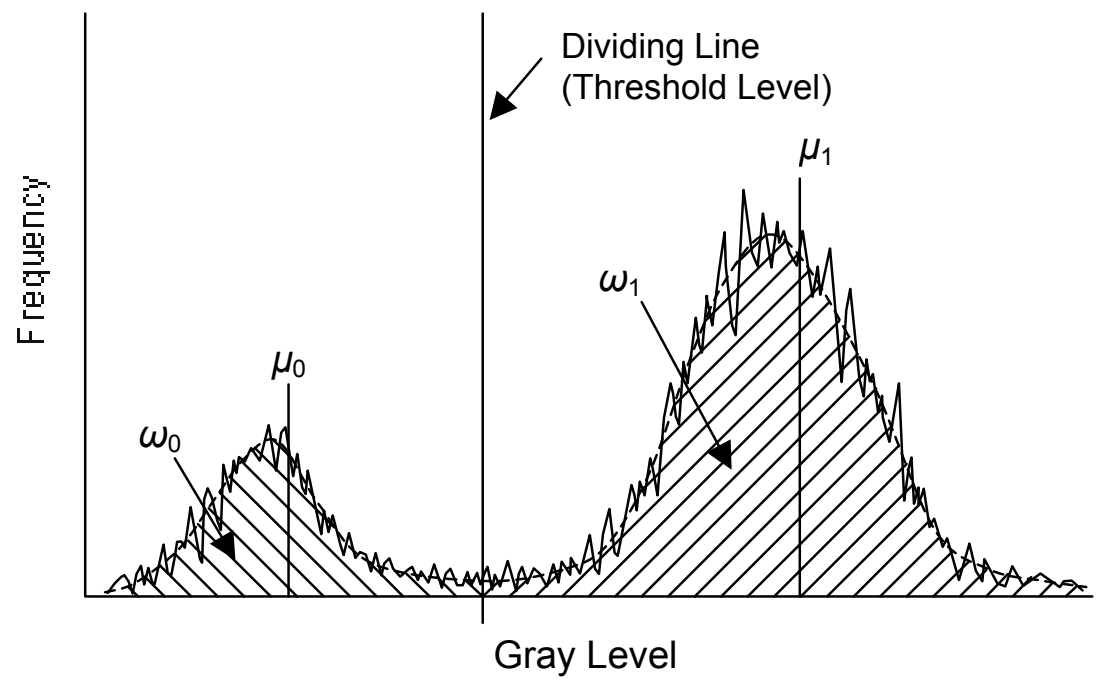

Fig. 13 Illustration of the method of Otsu (1979) for gray-level threshold

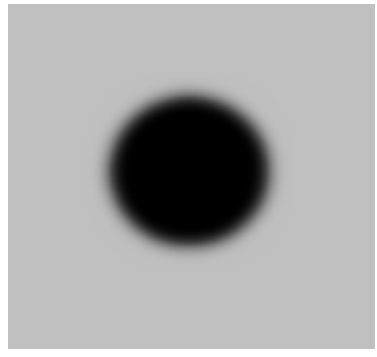

(a)

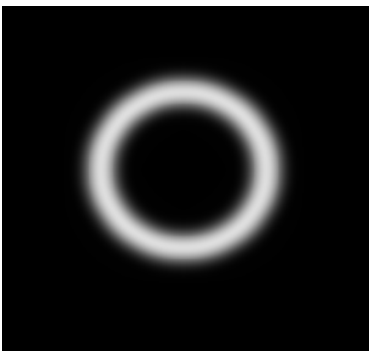

(c)

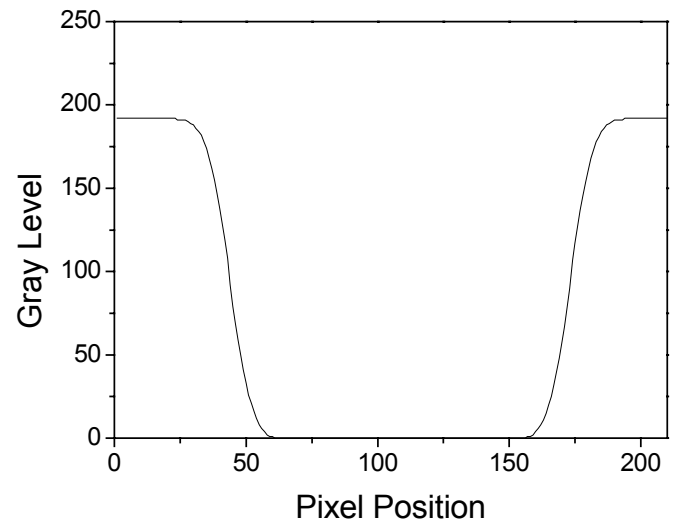

(b)

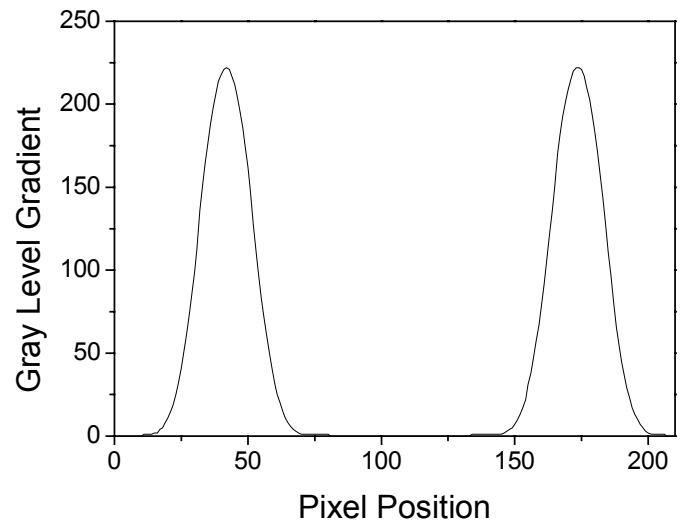

(d)

Fig. 14 Sample image and its gray level gradient 


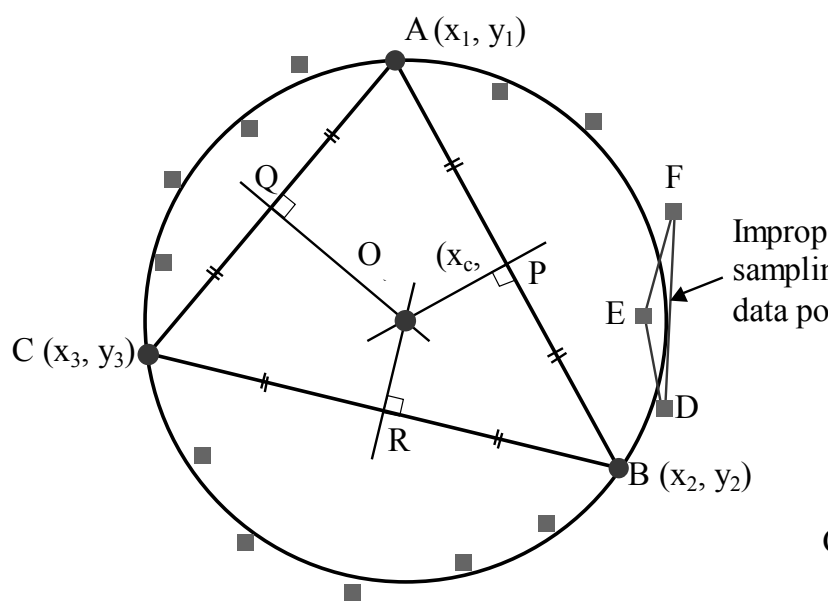

(a)

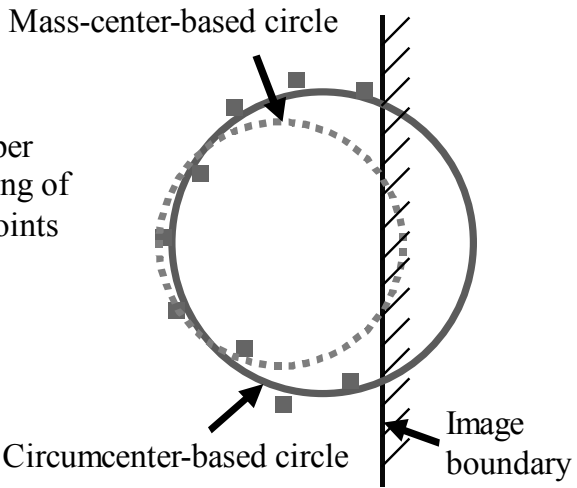

(b)

Fig. 15 Detection of the circumcenter [20] 


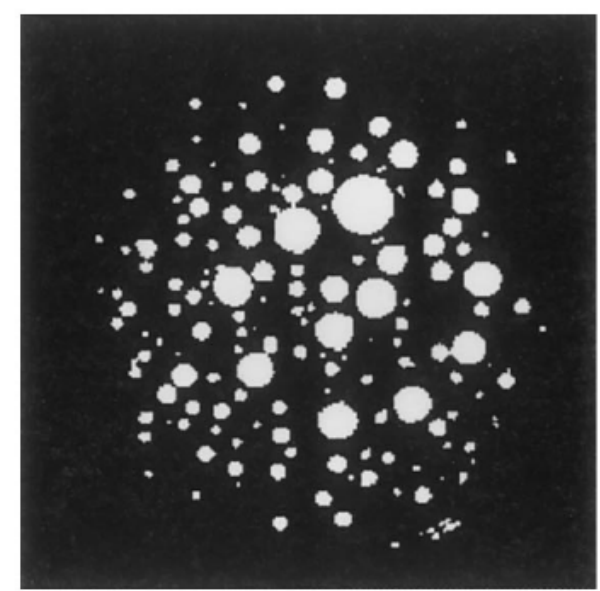

(a)

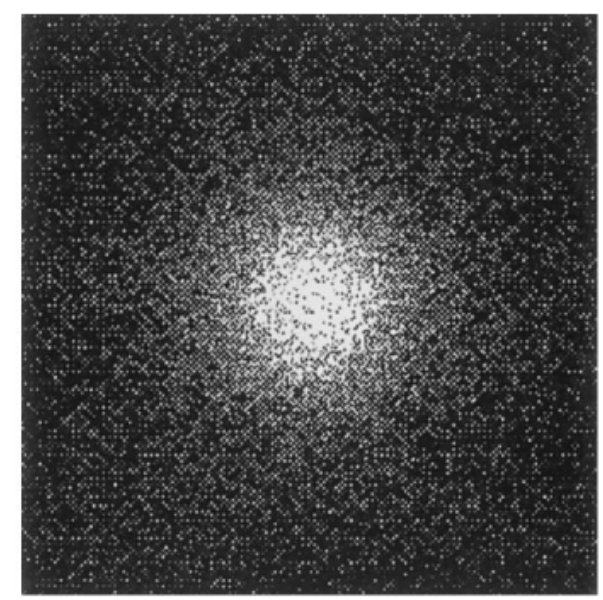

(c)

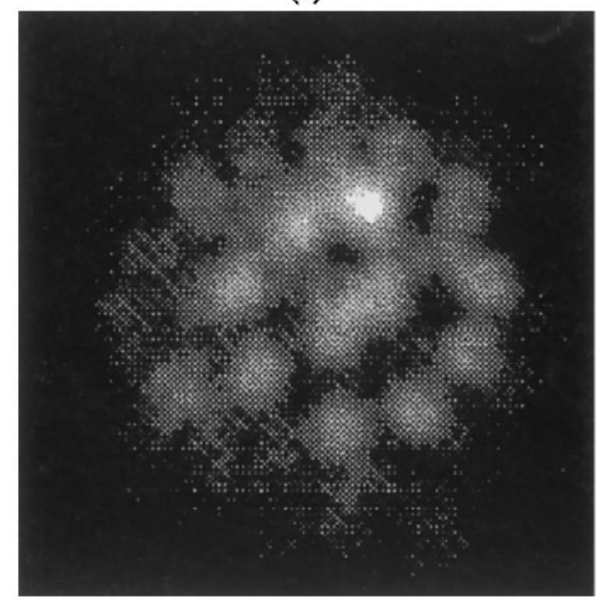

(e)

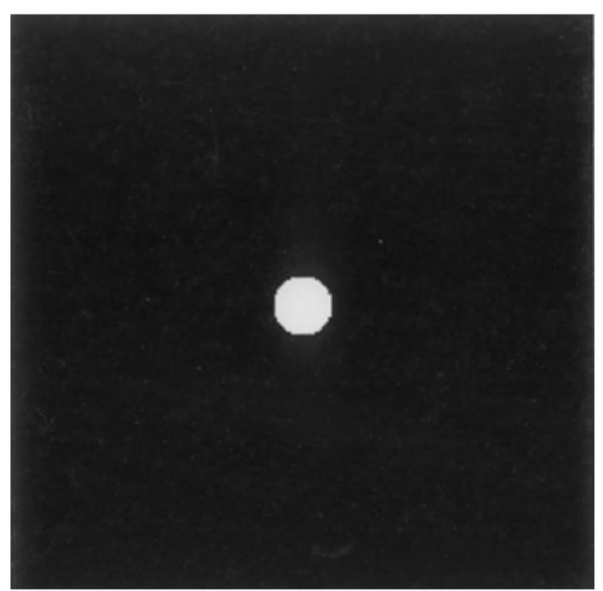

(b)

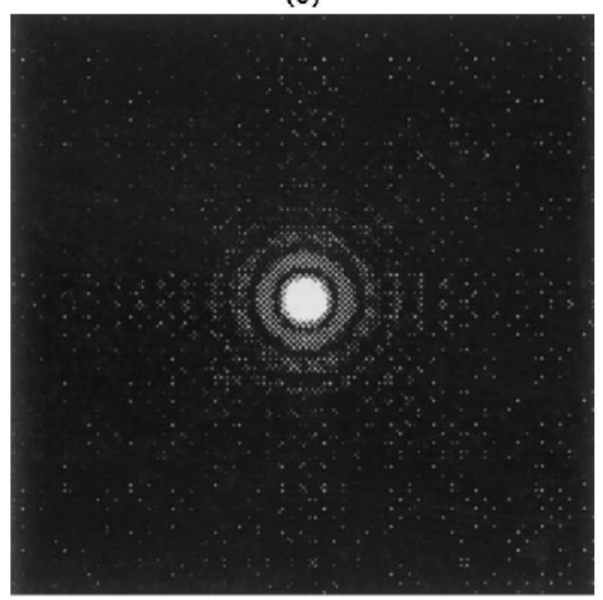

(d)

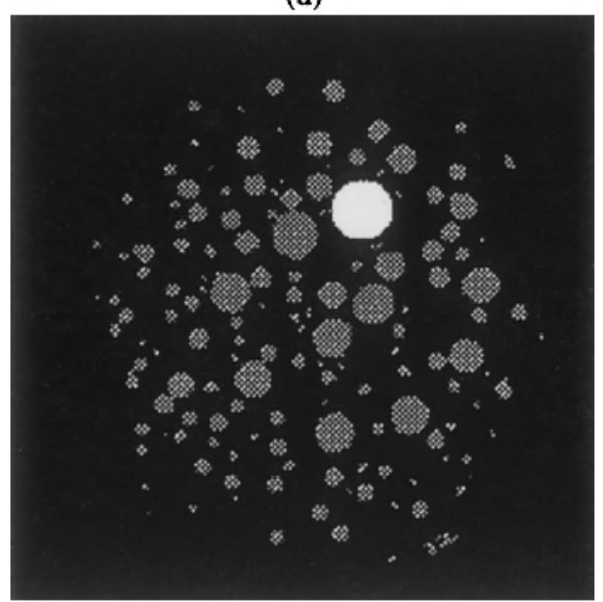

(f)

Fig. 16 Result of drop identification [17] 


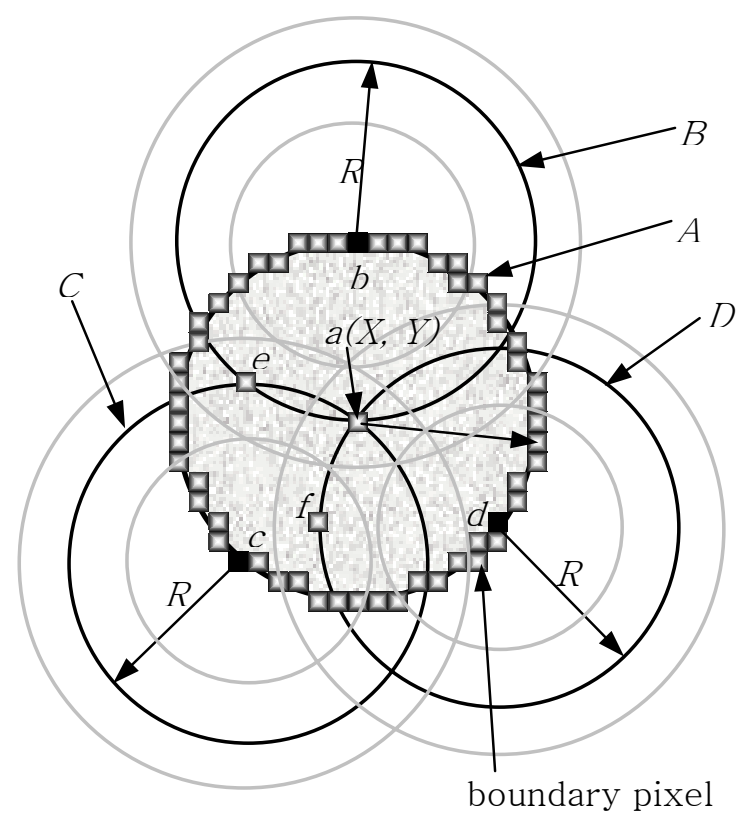

(a)

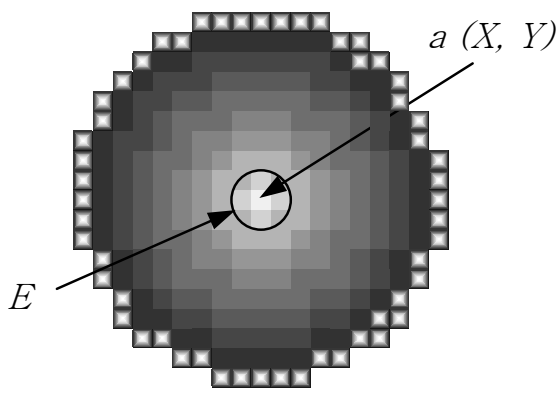

(b)

Fig. 17 Illustration of Hough transform

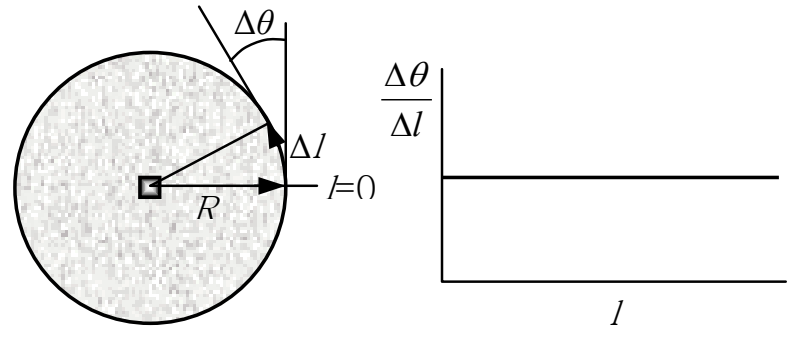

(a) Circle

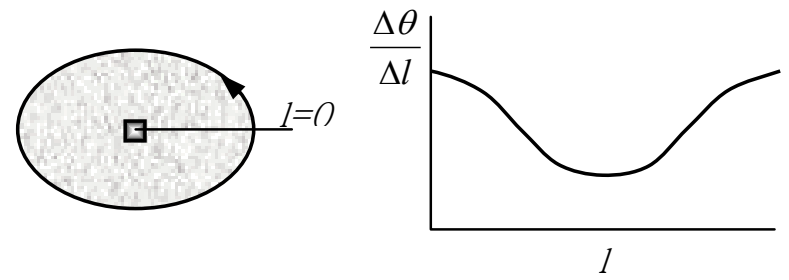

(b) Ellipse

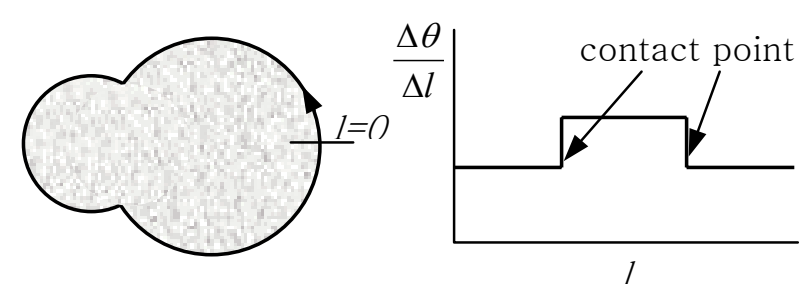

(c) Overlapped Particles

Fig. 18 Variation of boundary curvature [28] 


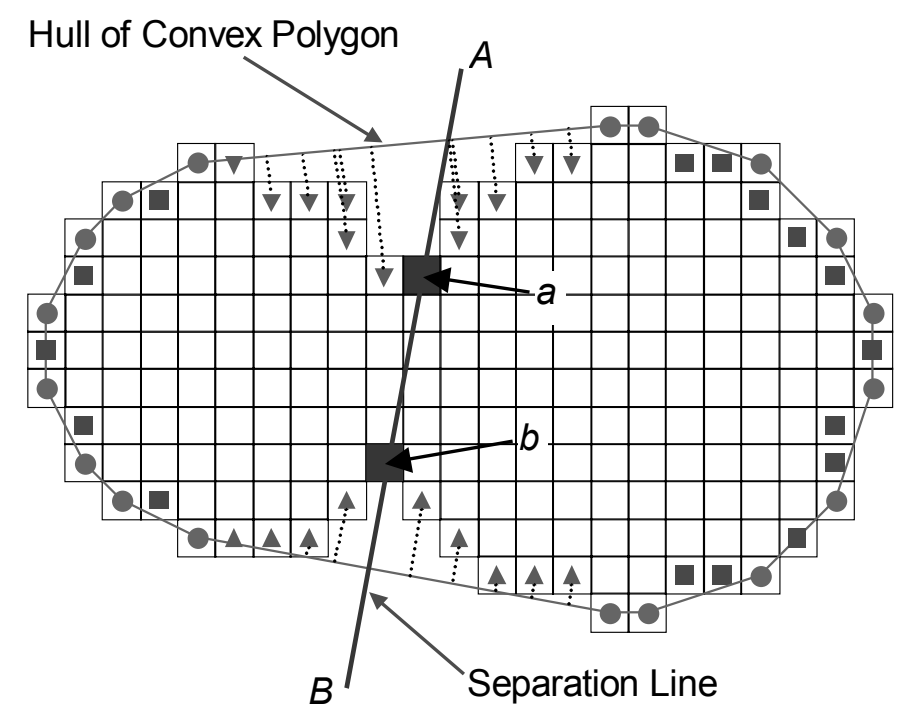

Fig. 19 Separation of an overlapped drop image

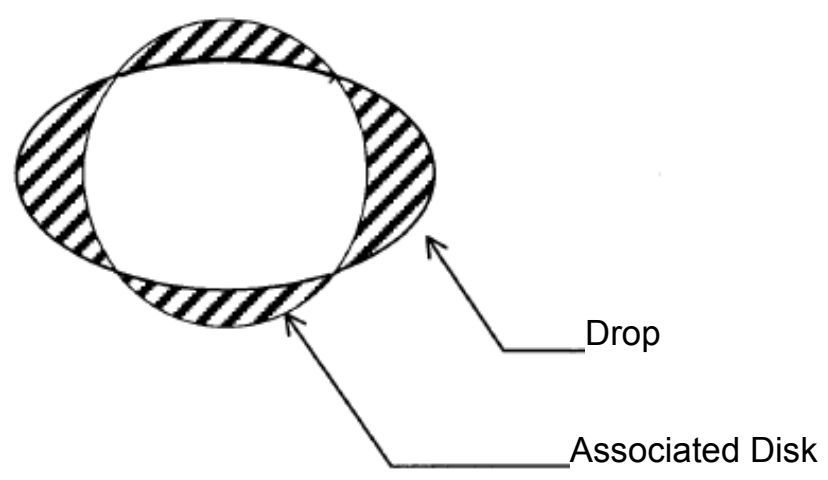

Fig. 20 Definition of shape parameter [4] 
Table 1 Overall algorithm of image processing

\begin{tabular}{|c|c|c|}
\hline & Steps/Description & Illustration \\
\hline & Global Process & \\
\hline 1 & $\begin{array}{l}\text { Boundary Detection in the Global Window } \\
\text { Gray level threshold using gray level histogram } \\
\text { Gray level gradient }\end{array}$ & \\
\hline 2 & $\begin{array}{l}\text { Circle Detection (Mass Center) } \\
\text { Center(x, y) and Radius }\end{array}$ & \\
\hline & Local Process & \\
\hline 3 & Local Window for Each Particle & \\
\hline 4 & $\begin{array}{l}\text { Boundary Detection in Local Window } \\
\text { Gray Level Threshold } \\
\text { Gray Level Gradient }\end{array}$ & \\
\hline 5 & $\begin{array}{l}\text { Separation of Overlapped Particles } \\
\text { Convex Hull, Hough Transform, Boundary } \\
\text { Curvature Algorithm }\end{array}$ & \\
\hline 6 & $\begin{array}{l}\text { Circle Detection } \\
\text { Circumcenter, Hough Transform, Boundary } \\
\text { Curvature Algorithm }\end{array}$ & \\
\hline 7 & $\begin{array}{l}\text { Out-of-Focus Particle Rejection } \\
\text { Gray Level Gradient, Value of Contrast, } \\
\text { Gray Level Gradient \& Value of Contrast }\end{array}$ & \\
\hline 8 & Depth-of-field Correction & \\
\hline 9 & Calculation of Mean Diameter & \\
\hline
\end{tabular}

